\title{
FutureJournal
}

\section{Uso de Sistema de Informação Geográfica na Gestão do Marketing Mix: o Caso de uma Rede de Calçados Femininos}

\author{
Rafaela Almeida Cordeiro \\ Doutoranda em Administração pela Escola Superior de Propaganda e Marketing, ESPM, Brasil \\ ralmeidacordeiro@gmail.com
}

\section{Denise Barbone}

Mestrado profissional em Comportamento do Consumidor pela Escola Superior de Propaganda e Marketing, ESPM, Brasil denise.barbone@gmail.com

\section{Ricardo Chagas Cruz}

Mestrado profissional em Comportamento do Consumidor pela Escola Superior de Propaganda e Marketing, ESPM, Brasil rcruz@espm.br

\section{Eduardo de Rezende Francisco}

Professor da FGV-EAESP e do Programa de Mestrado Profissional em Comportamento do Consumidor da ESPM eduardo.francisco@espm.br

\section{RESUMO}

Neste caso, estuda-se uma rede de lojas de calçado feminino (Rede A) que enfrenta problemas de estoque. A empresa não usa informações sobre clientes e localização do ponto de venda para renovar seu estoque, o que gera diminuição das vendas, excesso de produtos em algumas lojas e ruptura em outras, já que os modelos dos calçados adquiridos chegam a ser superiores (inferiores) à demanda. Assim, o objetivo do trabalho é mostrar como as ferramentas de Sistema de Informação Geográfica (SIG) podem ser aplicadas para solucionar o problema utilizando dados do Instituto Brasileiro de Geografia e Estatística (IBGE). Observou-se que a gestão do composto de marketing da Rede A não está alinhada ao seu público-alvo e potencial. A análise forneceu insights para ações a serem tomadas em relação ao mix de produtos das lojas, à precificação, à localização dos pontos de venda e ao posicionamento da marca. As contribuições das ferramentas de geomarketing e geoinformação são fundamentais para as decisões de varejo. A técnica de geoprocessamento permite analisar fenômenos e distribuições aplicadas ao marketing na medida em que auxiliam na identificação de padrões, além de utilizar, simultaneamente, inúmeros bancos de dados. Este trabalho contribui 


\section{FutureJournal}

Profuturo: Programa de Estudos do Futuro Editor Científico: James Terence Coulter Wright Avaliação: Double Blind Review, pelo SEER/OJS

para ampliar a discussão sobre as ferramentas SIG na área de marketing e para mostrar como ferramentas e dados gratuitos podem ser fortes aliados ao negócio.

PALAVRAS-CHAVE: Geomarketing. Marketing mix. Sistema de Informação Geográfica.

\section{Use of Geographic Information System in Marketing Mix Management: the Case of a Women's Footwear Network}

\section{ABSTRACT}

The case is based on a shoe store network (Store A) facing stock problems. The company does not use information on customers and location of the shops to renew its stock, which generates lower sales, excess or lack of products in some stores, since the shoes models available are higher (lower) than the demand. The objective of this study is to show how Geographic Information System (GIS) tools can be applied to solve the problem using the Instituto Brasileiro de Geografia e Estatística (IBGE) data. It was noted that Network A's marketing mix is not aligned to their target and potential audience. The analysis provides insights into actions to be taken in relation to the stores product mix, pricing, location of shops, and brand positioning. The contributions of geomarketing and geoinformation tools are critical to retail decisions. The GIS technique allows analyzing phenomena applied to marketing and distribution to the extent that assist in identifying patterns, in addition to use simultaneously several databases. This work helps to broaden the discussion of GIS tools in marketing and to show how free tools and data can be strong allies to business.

KEY-WORDS: Geomarketing. Marketing mix. Geographic Information System. 


\section{INTRODUÇÃO}

O comportamento do consumidor é um dos temas mais recorrentes na área de marketing, e tem como foco entender o processo decisório dos indivíduos em relação a produtos, serviços e experiências (Blackwell, Miniard \& Engel, 2011). Nesse contexto, surgem estratégias de segmentação de mercado para entender as diferenças dos consumidores em relação a necessidades, desejos e comportamentos de compra. Para tal, a empresa deve levar em consideração diferentes aspectos do seu público-alvo que afetam o processo de tomada de decisão, tais como fatores pessoais, sociais e culturais (Kotler \& Keller, 2006). Estudos mostram que características sociodemográficas, como escolaridade, renda e número de pessoas que compõem uma família, influenciam as atitudes dos consumidores em relação a produtos e marcas, e podem ser utilizadas para segmentar mercados (Wedel \& Kamakura, 2000; Kamakura \& Mazzon, 2013a). Portanto, deve-se considerar uma série de informações sobre o mercado e o consumidor para que a empresa obtenha bom desempenho.

Conhecer o cliente e suas características socioeconômicas permite uma melhor gestão do marketing mix, resultando em melhores decisões sobre tipo de produto ofertado, precificação, localização de loja, comunicação, tipos de promoção, entre outros. Uma das variáveis que têm permitido novas análises sobre o perfil de (potenciais) clientes e sobre as características de mercado (concorrência) é o avanço tecnológico. Se antes o dono da empresa era a maior fonte de informação para o negócio, hoje, é possível usar a tecnologia para construir modelos de previsão de vendas, desenvolver produto e até escolher a localização ideal para o ponto de venda (Machado, Francisco \& Ribeiro, 2006). Trata-se das ferramentas de Sistema de Informação Geográfica (SIG), que, ainda na década de 1990, já eram apontadas como o futuro do marketing de relacionamento, principalmente na tarefa de segmentação de mercado (Aranha, 1996), e muito têm contribuído para estratégias de marketing e de localização de varejistas (Baviera-Puig, Roig-Tierno, Buitrago-Vera \& Mas-Verdu, 2013).

No Brasil, alguns estudos mostram a aplicação das ferramentas SIG (também conhecidas como geomarketing e geoinformação) na localização de 
lojas de vestuário (Corujo, 2014); no mapeamento de clientes potenciais para uma rede de fast food (Prochnow, 2011); e na organização de equipes de venda de serviços de telecomunicação (Fagundes, Moriguchi, Vargas, Santana \& Sousa, 2009). Ainda assim, poucas empresas aproveitam o potencial dessas ferramentas na gestão estratégica, que, internacionalmente, têm maior aplicação no mercado varejista (ver Gijsbrechts, Campo \& Goossens, 2003).

O caso analisado neste trabalho refere-se a uma rede de lojas de calçados femininos que enfrenta problemas na montagem de seu estoque: a Rede A. A localização das lojas da Rede A foi definida com base em custo e potencial de consumo das regiões, no entanto, os resultados não atendem às expectativas e não trazem o retorno esperado. Os diretores não usam informações sobre clientes e localização do ponto de venda para renovar seu estoque, o que gera diminuição das vendas, excesso de estoque em algumas lojas e ruptura para outras, já que os modelos dos calçados adquiridos chegam a ser superiores (inferiores) à demanda de cada loja.

A partir da utilização de variáveis sociodemográficas disponíveis nas bases de dados do Instituto Brasileiro de Geografia e Estatística (IBGE), neste relato técnico, pretende-se mostrar como as ferramentas SIG podem solucionar o problema das vendas (e do estoque). A proposta de um mix assertivo para cada uma das quatro lojas foi elaborada com base na análise do mapa de vendas de cada loja da Rede A, na identificação do padrão de vendas dos modelos de calçados de cada loja e na utilização das ferramentas fornecidas pelo SIG. A contribuição do trabalho está em mostrar como o SIG pode ser uma ferramenta estratégica de gestão do marketing mix, ampliando as técnicas disponíveis no mercado para segmentação de cliente e gestão do negócio.

\section{CONTEXTO INVESTIGADO}

Fundada em 1955, no Rio Grande do Sul, a fornecedora de produtos da Rede A (Fornecedora A) nasceu com foco no público feminino. A partir dos anos 1980, a empresa passou a investir no aumento da sua capacidade produtiva, em pesquisas sobre tecnologia de conforto para calçados e na 
expansão do negócio para o exterior. A marca também passou por processo de extensão de linha e de marca, e lançou a sua loja virtual. Hoje, com 60 anos de existência, a empresa possui sete fábricas que produzem 50 mil pares de sapatos por dia, está presente em mais de 14 mil pontos de venda no Brasil, possui 26 lojas internacionais e exporta $30 \%$ da sua produção, marcando presença em 90 países.

A Fornecedora A posiciona-se como a marca do conforto, e toda a sua comunicação aborda esse atributo de produto. Os calçados são desenvolvidos com diferentes tecnologias que oferecem amortecimento, secagem de palmilhas, absorção de impactos, minimização de barulho, entre outros benefícios. Os produtos são desenvolvidos em P.U. italiano, que, além de ser uma matéria-prima ecologicamente sustentável, torna os calçados mais confortáveis e duráveis. As linhas de produto vão desde chinelos com saltos baixos e largos, a sandálias de festa, cujos saltos têm até 11 centímetros. Também há variedade de cores, modelos, tamanhos e estilos (passeio, festa ou trabalho). Com essa proposta de venda, a marca aposta não só no público maduro, mas também no infantojuvenil e adulto. A marca também disponibiliza uma revista online e um blog, em que as leitoras encontram dicas de saúde, tendências de moda, além de propostas de look com os produtos da marca.

Atualmente, a Fornecedora A, além de continuar disponibilizando seus produtos em diferentes lojas, aposta no conceito de loja exclusiva. São quatro lojas que oferecem, exclusivamente, os produtos desse fornecedor; as lojas encontram-se na cidade de São Paulo, em Moema; nos shoppings Tatuapé e SP Market; e uma loja no município de Barueri, no Shopping Tamboré. A proposta da Rede A é oferecer um mix de calçados que poderá ser encontrado apenas nas lojas exclusivas.

A Rede A é uma startup criada para atuar como varejo. Sua primeira parceira é a Fornecedora A, que lança quatro coleções por ano e na Rede A é possível encontrar mais de 200 modelos/cor por coleção, divididos em quatro linhas: trabalho, dia a dia, festa e conforto. As lojas possuem pisos amadeirados para que as consumidoras experimentem e testem os calçados, verificando os benefícios prometidos pelo produto, tais como conforto, maciez e alta absorção de impacto. As vendedoras recebem treinamento para 
entender o estilo de vida das consumidoras e para conhecer os atributos tecnológicos dos produtos; esse treinamento é dado por um gerente de produto da fábrica da Fornecedora A.

Os critérios de escolha para a localização das lojas da Rede A foram oportunidade de contrato (custo) e potencial de consumo das regiões. Não foi feita uma pesquisa de mercado para entender o perfil demográfico e socioeconômico das consumidoras em potencial, o que resultou em problemas de estoque. Considerando-se que os calçados têm preço médio de venda de $R \$ 120,00$, as lojas deveriam estar localizadas em regiões onde há maior concentração de pessoas das classes A e B, considerando-se, também, a idade média das moradoras de cada região de atuação.

\section{DIAGNÓSTICO DA SITUAÇÃO-PROBLEMA}

A Rede A tem apresentado problemas no resultado de suas vendas em função da montagem de seu estoque e da localização de suas lojas. Para solucionar a situação, a empresa contratou uma consultora especialista em varejo, cuja expertise e cujo acesso às informações da loja foram utilizados na elaboração deste trabalho. Uma das primeiras análises foi identificar que o padrão de venda das lojas varia de acordo com as linhas de produto, a saber: trabalho, dia a dia, festa e conforto. Além disso, observou-se que algumas lojas estão geograficamente muito próximas ou em regiões onde não há potencial de consumo para os produtos ofertados. A partir das primeiras observações, as demais análises foram realizadas: levantamento de vendas por loja e por linha de produto, mapeamento geográfico das lojas e análise do potencial de consumo de cada região.

O faturamento da empresa, apresentado no Gráfico 1 e na Tabela 1, mostra que a loja de Moema, embora possua o maior estoque, é a que tem menor faturamento. Isto se deve a três características: i) é nessa loja que fica o estoque central da rede; ii) o mix de produtos oferecido na loja não condiz com a demanda da região onde está localizada; iii) a loja está localizada em frente ao Shopping Ibirapuera, concorrente direto (no período em que o trabalho estava sendo realizado, a loja da Rede A de Moema - antes localizada em uma rua - foi transferida para o Shopping Ibirapuera. Esta 
decisão foi baseada no trabalho de consultoria prestado por um dos autores deste relato).

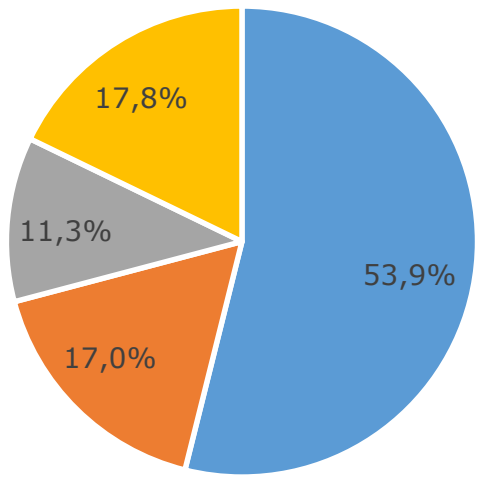

- Moema - Shopping Tatuapé - Shopping Tamboré - Shopping SP Market

Gráfico 1: Participação de cada loja (\%) no estoque de produtos

Tabela 1: Faturamento por loja (maio/2016)

\begin{tabular}{c|c|c|c|c}
\hline Moema & $\begin{array}{c}\text { Shopping } \\
\text { Tatuapé }\end{array}$ & $\begin{array}{c}\text { Shopping } \\
\text { Tamboré }\end{array}$ & $\begin{array}{c}\text { Shopping SP } \\
\text { Market }\end{array}$ & Total \\
\hline $\mathrm{R} \$ 33.386,46$ & $\mathrm{R} \$ 85.682,80$ & $\mathrm{R} \$ 42.760,00$ & $\mathrm{R} \$ 79.659,45$ & $\mathrm{R} \$ 241.488,71$ \\
\hline
\end{tabular}

As lojas dos shoppings SP Market e Tatuapé são as que apresentam maior faturamento e ticket médio (para todas as linhas de produto), como pode ser observado nas Tabelas 1 e 2 . Essas lojas também apresentam o maior preço médio por produto. O mesmo comportamento não pode ser observado para a loja do shopping Tamboré, que tem o menor faturamento e o menor ticket médio.

Tabela 2: Ticket médio loja X produto (maio/2016)

\begin{tabular}{l|c|c|c|c}
\hline $\begin{array}{l}\text { Tipo de } \\
\text { calçado }\end{array}$ & Moema & $\begin{array}{c}\text { Shopping } \\
\text { Tatuapé }\end{array}$ & $\begin{array}{c}\text { Shopping } \\
\text { Tamboré }\end{array}$ & $\begin{array}{c}\text { Shopping } \\
\text { SP Market }\end{array}$ \\
\hline Bota & $\mathrm{R} \$ 189,01$ & $\mathrm{R} \$ 195,28$ & $\mathrm{R} \$ 173,83$ & $\mathrm{R} \$ 196,29$ \\
\hline Chinelo & $\mathrm{R} \$ 71,30$ & $\mathrm{R} \$ 84,53$ & $\mathrm{R} \$ 74,09$ & $\mathrm{R} \$ 84,33$ \\
\hline Sandália & $\mathrm{R} \$ 115,09$ & $\mathrm{R} \$ 134,07$ & $\mathrm{R} \$ 122,40$ & $\mathrm{R} \$ 132,23$ \\
\hline Sapato & $\mathrm{R} \$ 126,22$ & $\mathrm{R} \$ 142,44$ & $\mathrm{R} \$ 130,30$ & $\mathrm{R} \$ 141,44$ \\
\hline Tamanco & $\mathrm{R} \$ 94,07$ & $\mathrm{R} \$ 108,84$ & $\mathrm{R} \$ 105,22$ & $\mathrm{R} \$ 111,21$ \\
\hline Tênis & $\mathrm{R} \$ 139,90$ & $\mathrm{R} \$ 147,76$ & $\mathrm{R} \$ 132,90$ & $\mathrm{R} \$ 148,96$ \\
\hline
\end{tabular}


Nas Tabelas 2 e 3, apresentam-se os resultados de vendas por produto em cada loja. Considerando-se as características dos calçados, como tipo de salto (alto, médio, baixo) e tecnologias, é possível associar as linhas de produto (trabalho, dia a dia, festa e conforto) ao perfil de consumo de cada loja da rede. Essa análise foi feita para solução do problema e proposta de intervenção.

Tabela 3: Número de produtos vendidos loja $X$ linha (maio/2016)

\begin{tabular}{l|c|c|c|c}
\hline $\begin{array}{l}\text { Linha de } \\
\text { produto }\end{array}$ & Moema & $\begin{array}{c}\text { Shopping } \\
\text { Tatuapé }\end{array}$ & $\begin{array}{c}\text { Shopping } \\
\text { Tamboré }\end{array}$ & $\begin{array}{c}\text { Shopping } \\
\text { SP Market }\end{array}$ \\
\hline Trabalho & 50 & 260 & 139 & 148 \\
\hline Dia a dia & 68 & 170 & 62 & 318 \\
\hline Festa & 0 & 49 & 14 & 30 \\
\hline Conforto & 93 & 70 & 15 & 15 \\
\hline
\end{tabular}

Observa-se que a loja de Moema tem a maior venda de produtos da linha conforto; as lojas dos shoppings Tatuapé e Tamboré apresentam maior venda de calçados da linha trabalho; a loja do shopping SP Market tem maior venda de produtos da linha dia a dia. Essa observação revela que cada loja deveria ter um mix de produtos alinhado à demanda, evitando problemas de estoque e baixo retorno de vendas.

\section{INTERVENÇÃO PROPOSTA}

Foi observado que a gestão do composto de marketing das lojas da Rede A parece não estar alinhada ao seu público-alvo e potencial. Por meio das ferramentas SIG (ArcView), foram utilizados dados do IBGE para mapear as regiões onde as lojas estão localizadas, considerando-se as variáveis idade da população feminina e renda média da população. Esses dados foram cruzados com as informações fornecidas pela loja para gerar a proposta de intervenção.

As ferramentas de geomarketing e geoinformação estão sendo, cada vez mais, adotadas por indivíduos, empresa, governos e sociedades, com os mais diversos objetivos: mobilidade, redução de custos, segurança, geração de conteúdo, entre outros. As ferramentas SIG "combinam informações e dados demográficos, socioeconômicos e de negócios com recursos de 
mapeamento e visualização gráfica" (Miller, Holmes \& Mangold, 2007, p. 49, tradução nossa). Vários exemplos práticos podem ser apresentados: a empresa de logística UPS reduziu os custos com combustíveis e otimizou a organização de equipes de trabalho com a utilização de ferramentas SIG; o governo de Sierra Nevada, no México, realizou um mapeamento participativo da região (incluindo o trabalho da comunidade, de universidades e de empresas) para desenvolver e implementar um projeto de sustentabilidade (Pozzebon \& Delgado, 2012).

Essas ferramentas são amplamente aplicadas às estratégias de marketing quanto à localização de loja, campanhas publicitárias regionais e atividades de marketing direto, além de qualidade do serviço e satisfação do cliente (Baviera-Puig et al., 2013; Mittal, Kamakura \& Govind, 2004; Ozimec, Natter \& Reutterer, 2010). Tais tecnologias têm permitido realizar análises de mercado por meio da visualização de mapas, o que facilita o entendimento de gestores e muda a maneira como as empresas gerenciam e utilizam as informações sobre os seus clientes (Nunes, Santana, Bezerra \& Sobral, 2014).

Considerando-se que a localização é o mecanismo para equilibrar oportunidade de mercado, oferta e demanda (Thompson, 2010), as ferramentas SIG têm desempenhado um papel importante no sucesso das empresas. Com inspiração nessas informações, a solução para a Rede $A$ foi desenvolvida com base nas ferramentas SIG e nos dados do Censo Demográfico de 2010 do IBGE. A seguir, são apresentadas as principais análises. 


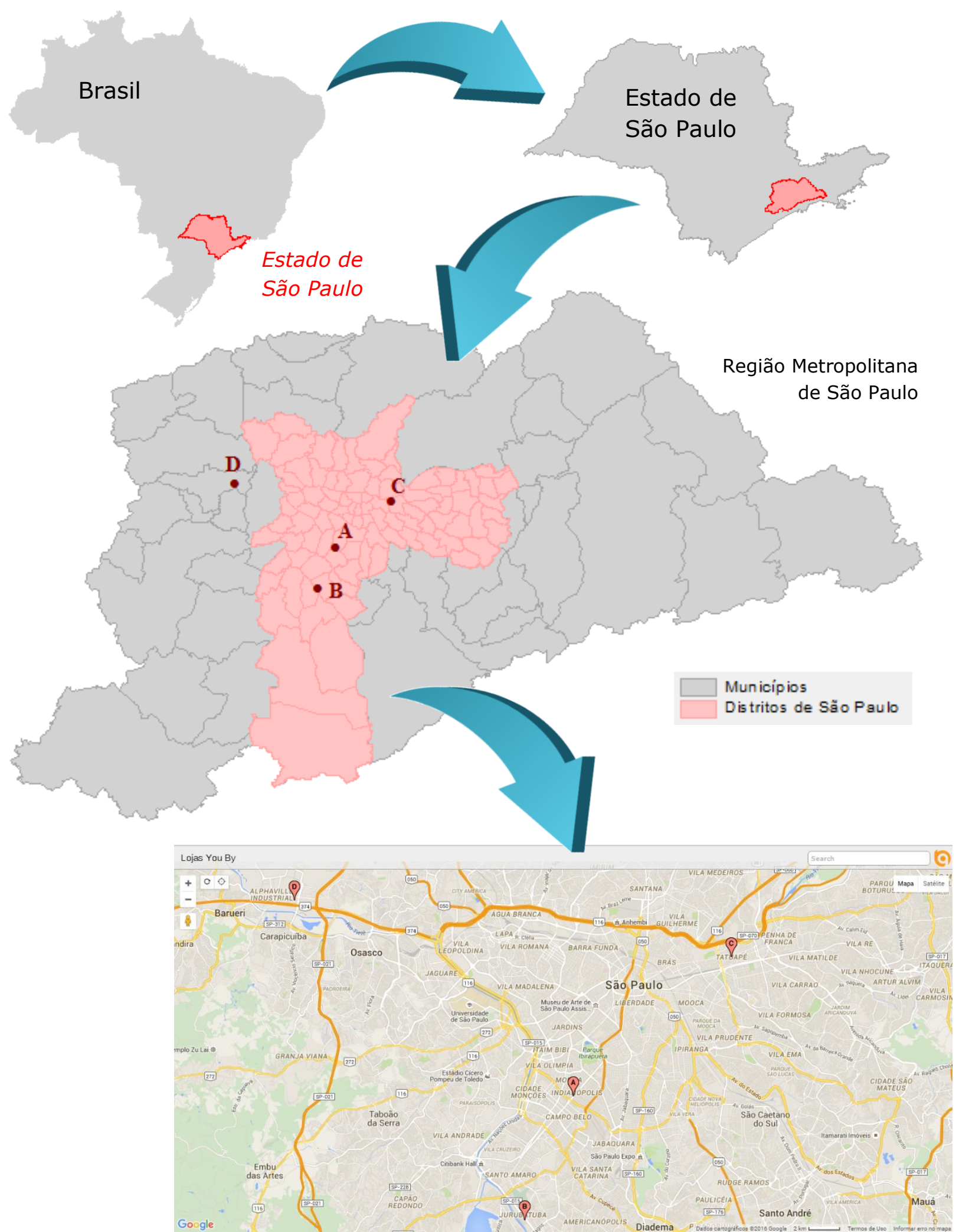

Nota: Loja A - Moema; Loja B - Shopping SP Market; Loja C - Shopping Tatuapé; Loja D - Shopping Tamboré.

Figura 1: Mapa de localização das lojas da Rede A em São Paulo, Brasil 


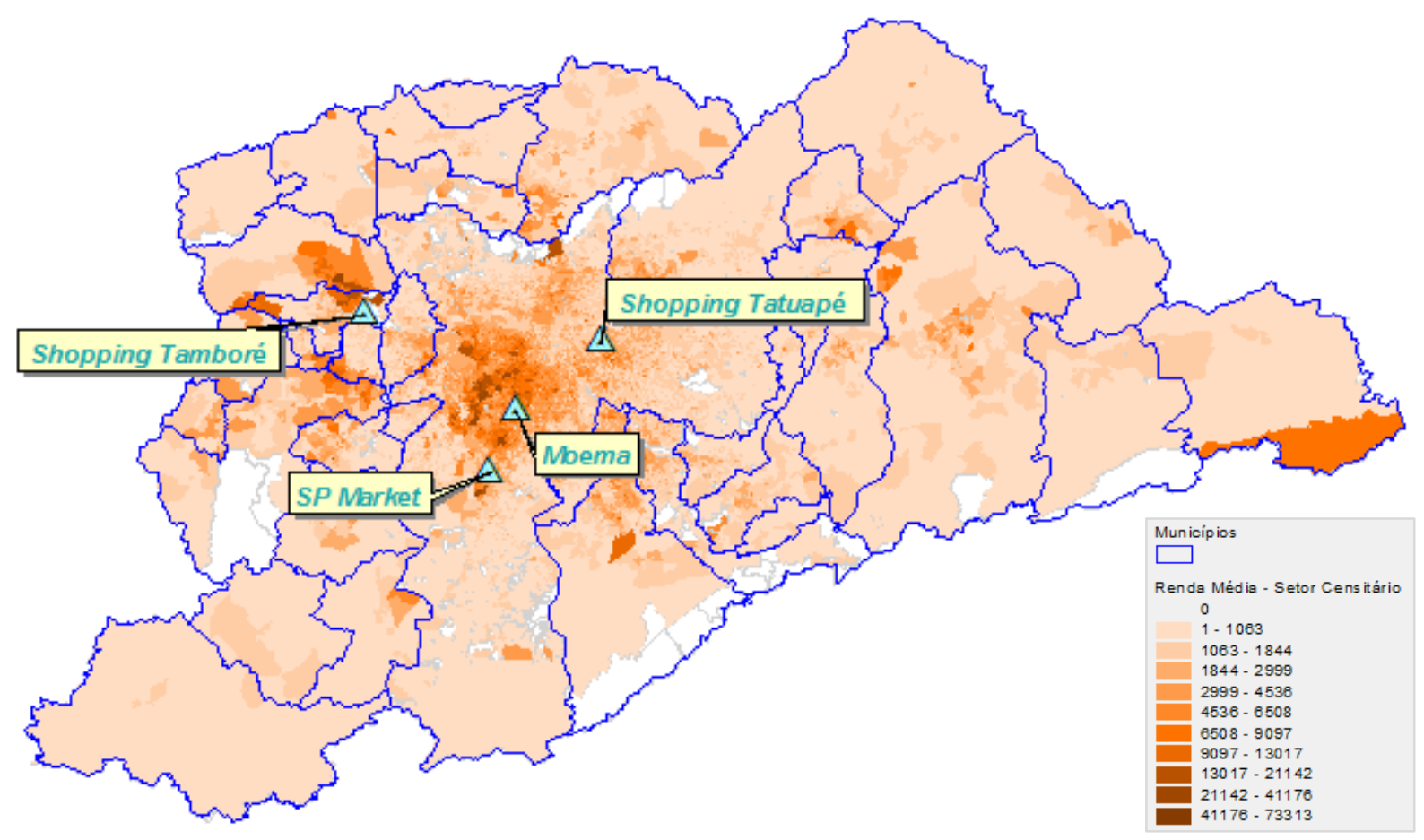

Figura 2: Mapa das lojas da Rede A e da distribuição de renda por setor censitário na região metropolitana de São Paulo

Fonte: IBGE (2010) 
Moema

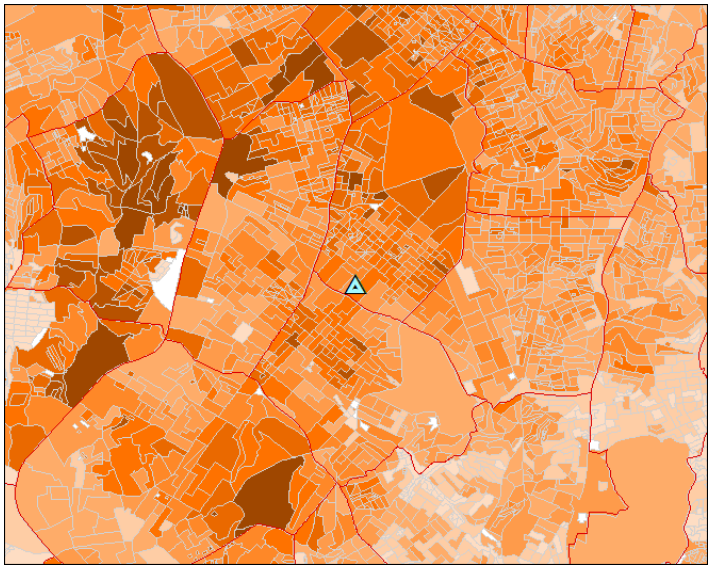

Shopping Tatuapé

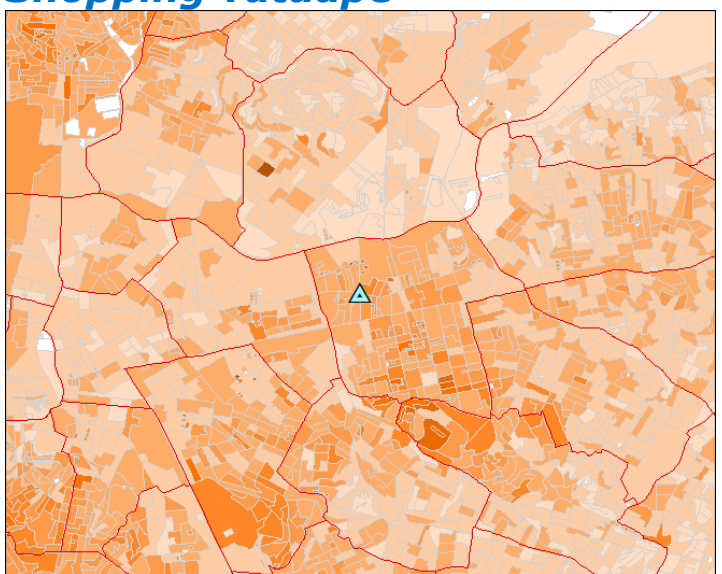

SP Market

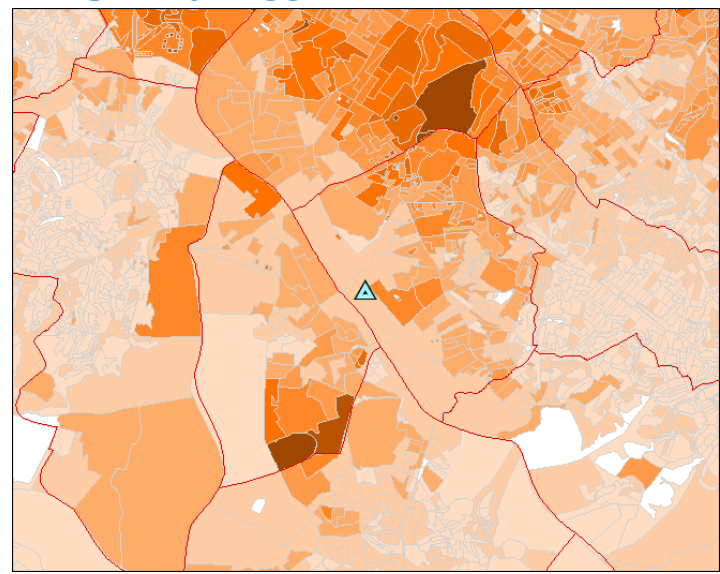

Shopping Tamboré

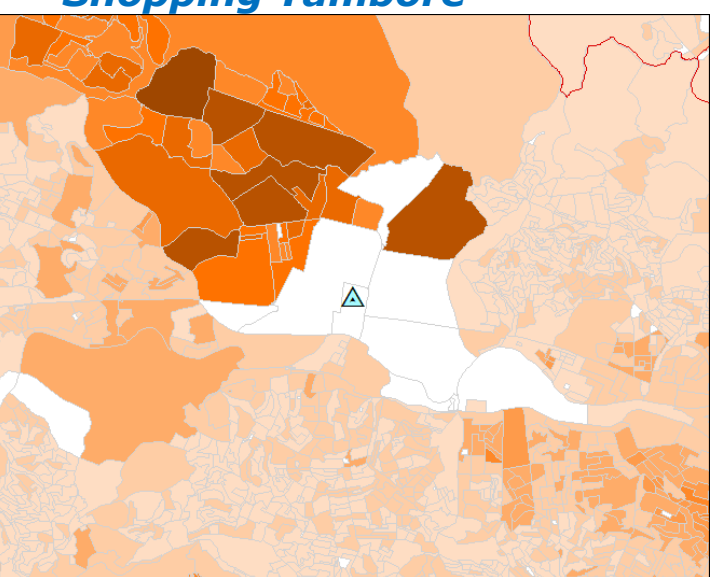

Legenda:

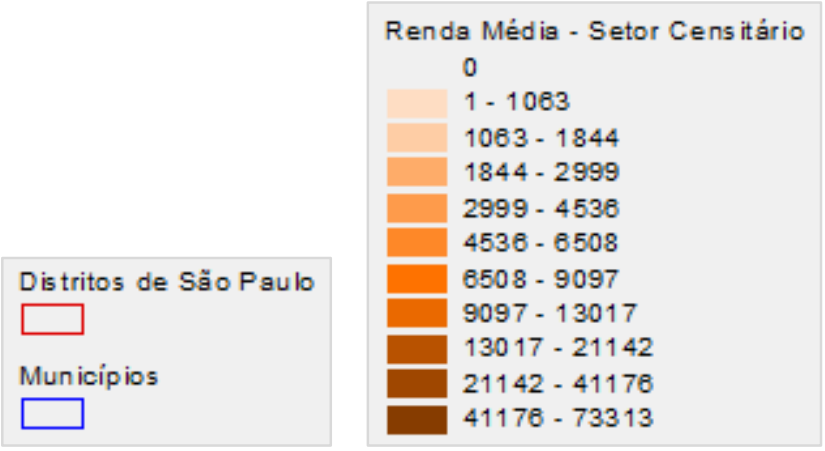

Nota: Setores censitários em branco nos mapas correspondem a áreas não residenciais. Notadamente, a região em que o Shopping Tamboré está localizado apresenta essa característica.

Figura 3: Mapa das lojas da Rede A e da distribuição de renda por setor censitário na região metropolitana de São Paulo - aproximação (zoom) por loja

Fonte: IBGE (2010)

As regiões onde as lojas estão localizadas apresentam as seguintes características, segundo o Censo Demográfico do IBGE (2010): 
i) Distrito de Moema (loja Moema): maior concentração de famílias nas faixas de renda média entre $\mathrm{R} \$ 4.000$ e $\mathrm{R} \$ 14.000$, com algumas áreas onde há maior concentração de pessoas na faixa até $\mathrm{R} \$ 25.000$. A renda per capita do distrito é $\mathrm{R} \$ 4.967,83$.

ii) Distrito de Campo Grande (loja Shopping SP Market): maior concentração de famílias nas faixas de renda média até $\mathrm{R} \$ 2.800$, com algumas áreas onde predomina população na faixa até $\mathrm{R} \$ 7.200$. A renda per capita é $\mathrm{R} \$ 1.769,23$.

iii) Distrito de Tatuapé (loja Shopping Tatuapé): maior concentração de famílias nas faixas de renda média até $\mathrm{R} \$ 5.500$, com algumas áreas onde há maior concentração de pessoas na faixa até $\mathrm{R} \$ 9.700$. A renda per capita é $\mathrm{R} \$ 2.158,82$.

iv) Município de Barueri (loja Shopping Tamboré): loja em região de maior concentração de famílias nas faixas de renda média entre $R \$$ 4.500 e R\$21.000. Notadamente, como contraponto, a renda per capita do município é $\mathrm{R} \$ 877,46$.

As informações apresentadas no Gráfico 2 mostram a renda per capita da população de cada um dos distritos analisados.

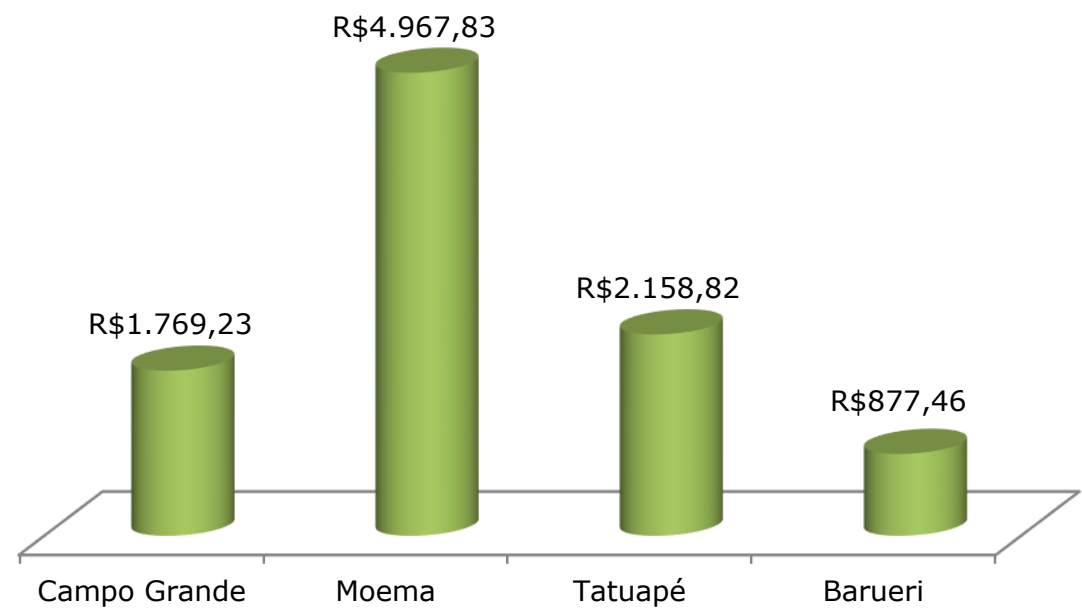

\section{Gráfico 2: Renda per capita dos distritos ou município onde as lojas estão localizadas}

Fonte: IBGE (2010, citado por Fundação Seade, s/d)

Sabendo-se que a renda é uma das variáveis que mais contribuem para diferenças significativas de consumo e têm impacto direto na capacidade do consumidor em adquirir e substituir produtos (Kamakura \& Mazzon, 
2013b), percebe-se que o problema de desempenho das lojas não é causado apenas pela localização. Embora o distrito de Moema apresente a maior renda per capita, a loja tem o pior desempenho. Acredita-se que uma possível causa seja a localização (uma loja de rua) e o mix de produtos.

Considerando-se a população dos distritos, foi feita uma análise da população feminina: Moema tem 71.459 habitantes, com predominância de mulheres na faixa etária de 30 a 59 anos; é o distrito que apresenta o maior número de mulheres entre 60 a 74 anos e a maior taxa de envelhecimento. O distrito de Campo Grande possui 96.756 habitantes, com a maioria das mulheres na faixa etária de 30 a 59 anos; é o distrito (entre os demais investigados na cidade de São Paulo) que apresenta maior número de mulheres entre 20 a 29 anos. O distrito de Tatuapé tem uma população de 75.481 habitantes; a maioria das mulheres residentes encontra-se na faixa etária de 30 a 59 anos; é o distrito com maior número de mulheres com 75 anos ou mais, e a segunda maior taxa de envelhecimento. O município de Barueri tem 240.656 habitantes, com a população feminina predominantemente na faixa etária de 30 a 44 anos; é a região que apresenta o menor número de mulheres com 75 anos ou mais e a menor taxa de envelhecimento. Os dados podem ser observados nos Gráficos 3 e 4.

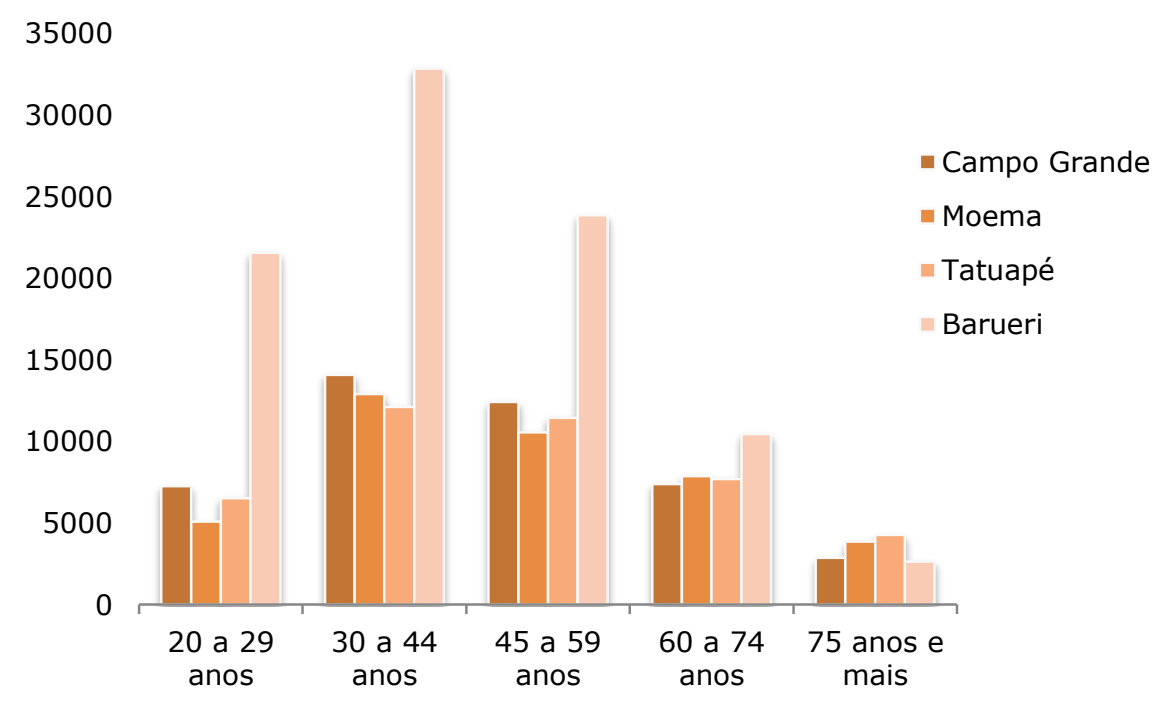

Gráfico 3: População feminina por faixa etária 


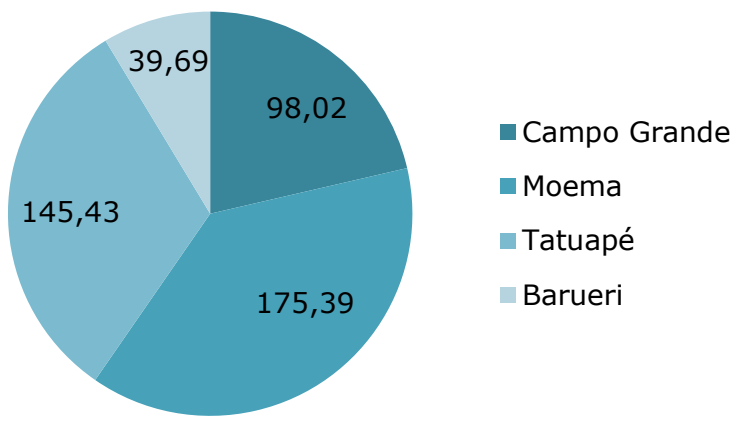

Nota: taxa de envelhecimento significa a proporção de pessoas de 60 anos e mais por 100 indivíduos de 0 a 14 anos.

\section{Gráfico 4: Taxa de envelhecimento (\%)}

Fonte: IBGE (2010, citado por Fundação Seade, s/d)

As regiões de Moema e Tatuapé possuem a população feminina com idade mais avançada, o que sugere que essas lojas devem focar um mix de produtos das linhas dia a dia e conforto. Além disso, as duas regiões apresentam as maiores taxas de envelhecimento e as maiores rendas. $\mathrm{O}$ distrito de Campo Grande, onde está localizada a loja do Shopping SP Market, é caracterizado por uma população feminina mais jovem e de renda mais baixa. Sendo assim, sugere-se um estoque maior de calçados da linha trabalho (o mais vendido), festa e dia a dia, com preços mais acessíveis.

A loja de Barueri, localizada no Shopping Tamboré, requer uma análise mais detalhada. O município apresenta a menor renda per capita, no entanto, trata-se de uma região comercial onde estão localizadas empresas de grande porte (Walmart, Tok\&Stok, entre outras) - vizinha de domicílios de alto poder aquisitivo. Isso sugere que o Shopping Tamboré é utilizado como ponto de apoio para almoço e happy hours, atraindo executivos que trabalham nas imediações, podendo implicar um mix de calçados que atenda às mulheres que moram e às mulheres que trabalham nessa região. Portanto, o estoque de produtos deveria focar as linhas trabalho e dia a dia, com preços mais acessíveis.

Pensando no crescimento do negócio, foi realizada uma análise sobre potenciais regiões para a abertura de novas lojas da Rede A. Com base na distribuição de renda e na população feminina, sugere-se que uma nova loja seja inaugurada em um dos distritos de Jardim Paulista ou Pinheiros. Ambos 
são caracterizados pela alta concentração de renda e pela maioria das mulheres na faixa dos 30 aos 44 anos. Os dados podem ser observados nos Gráficos 5 e 6.

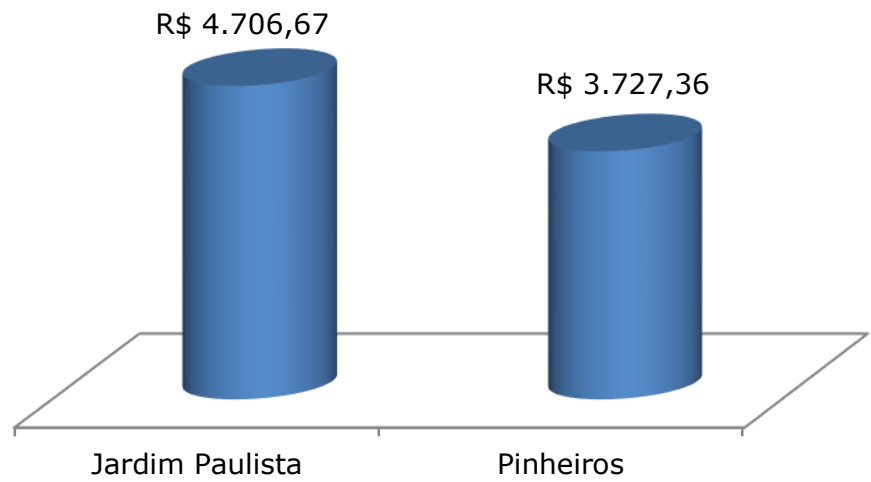

\section{Gráfico 5: Renda per capita}

Fonte: IBGE (2010, citado por Fundação Seade, s/d)

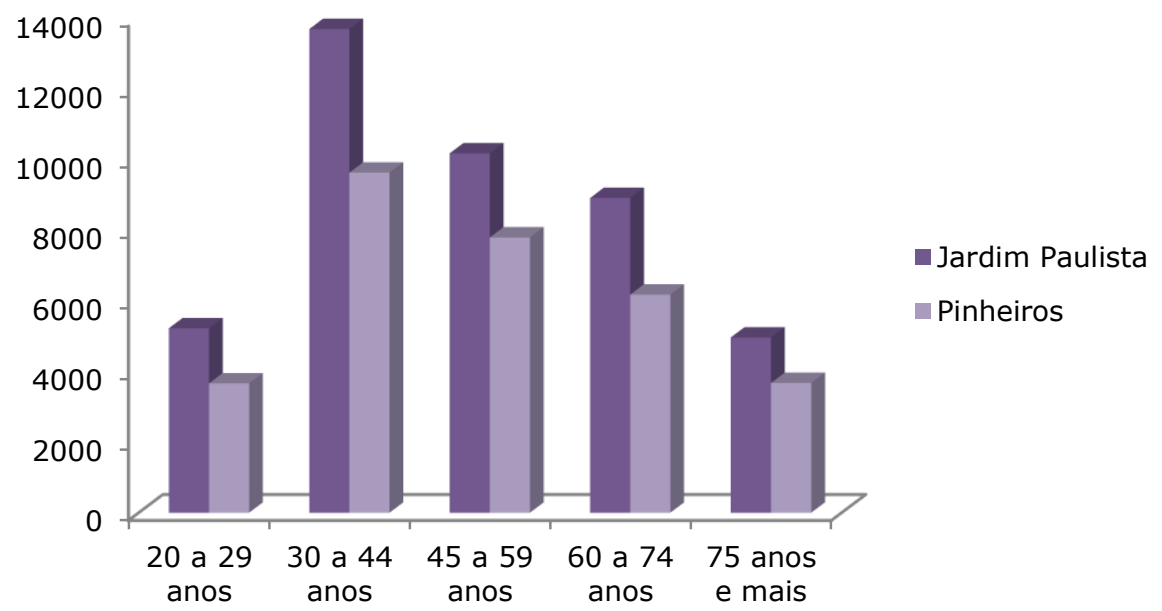

\section{Gráfico 6: População feminina}

Fonte: IBGE (2010, citado por Fundação Seade, s/d) 


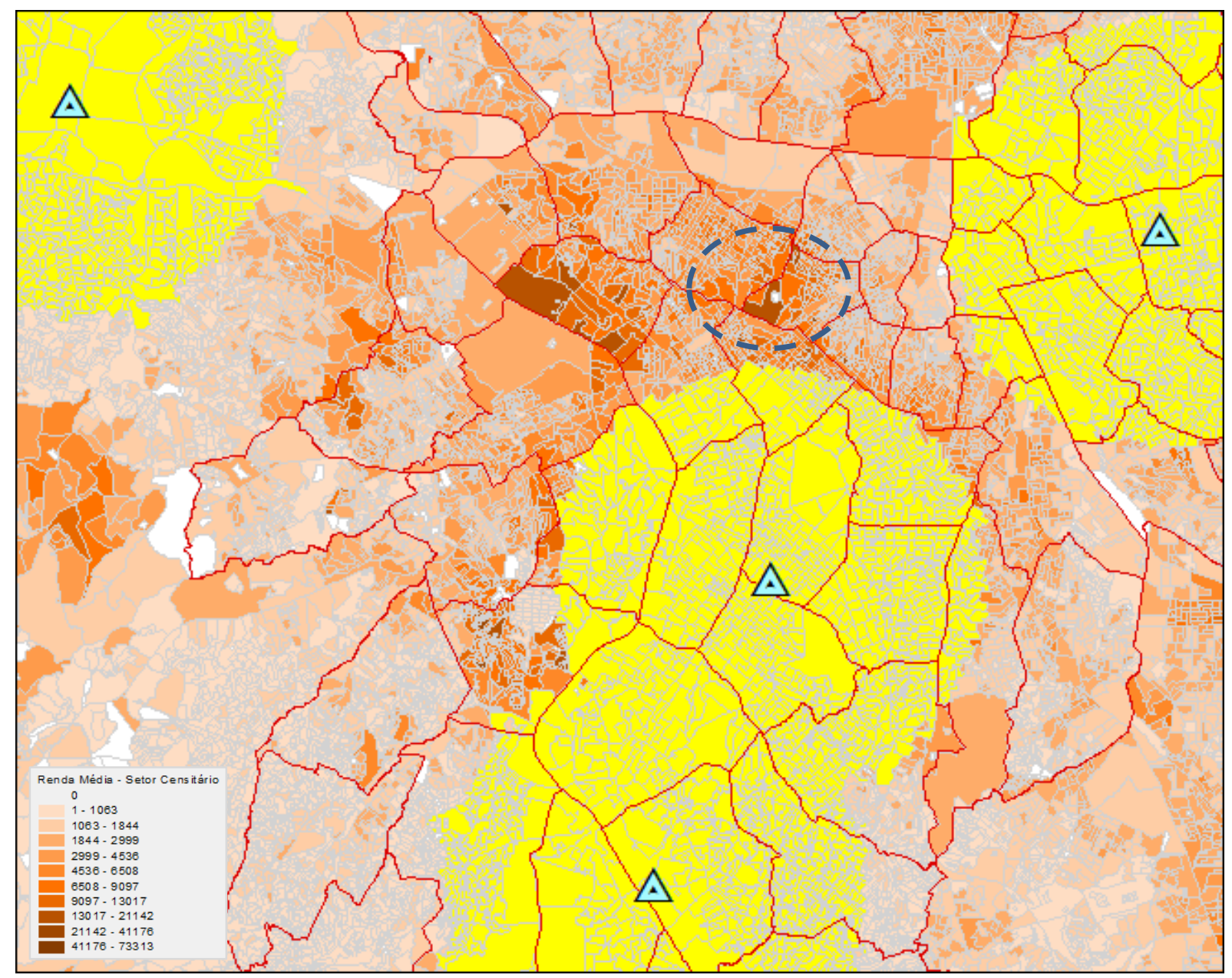

Nota: As regiões em amarelo estão no entorno de até cinco quilômetros das atuais lojas da Rede A.

Figura 4: Região para abertura de uma nova loja da Rede A Fonte: IBGE (2010)

$\mathrm{Na}$ Figura 4, apresentam-se, em amarelo, as regiões que compreendem o entorno de até cinco quilômetros de cada uma das lojas da Rede A. Com base no público-alvo anteriormente discutido, vislumbra-se a oportunidade de atuação na região destacada no mapa (elipse pontilhada).

As informações apresentadas oferecem um olhar estratégico de marketing que vai além das análises e sugestões de localização e estoque das lojas. Considerando as linhas de produto da Fornecedora A, o preço praticado e as regiões de atuação, sugere-se que a Rede $A$ reveja seu posicionamento. Acredita-se que o foco deva ser o público feminino que está na faixa etária de 40 anos ou mais: são mulheres que ainda estão no mercado de trabalho, mas que prezam pelo conforto (que é a proposta de venda da marca). Esse público, geralmente, tem renda mais elevada e costuma ir aos shoppings onde as lojas estão localizadas, os quais, de acordo com a 
Associação Brasileira de Shopping Centers, são frequentados, predominantemente, pelo público de classe B.

\section{RESULTADOS OBTIDOS}

O uso das ferramentas SIG no caso da Rede A mostrou como a tecnologia pode ser aplicada à estratégia do negócio. A análise das regiões onde as lojas estão localizadas forneceu insights para ações a serem tomadas em relação ao mix de produtos das lojas (produto), à precificação (preço), à localização dos pontos de venda (praça) e ao posicionamento da marca. As análises permitem que os gestores gerenciem melhor seus estoques de loja considerando as características de suas (potenciais) clientes e da região de atuação, o que resulta em melhor desempenho de mercado e maior retorno.

No mercado internacional, mais de $50 \%$ dos varejistas e comerciantes utilizam as ferramentas SIG na tomada de decisão de marketing (Ozimec et al., 2010). Quando o assunto é localização do ponto de venda, Thompson (2010) argumenta que o sucesso do negócio é totalmente dependente da sua localização; sendo esta uma decisão acertada, é possível configurar o estoque de mercadoria com base no perfil dos consumidores da região e de suas necessidades, aumentando o retorno sobre o investimento e o lucro.

Este estudo ainda permitiu a indicação de uma potencial localidade para abertura de uma nova loja. As características demográficas e socioeconômicas da população cruzadas com as informações sobre os produtos da loja permitiram identificar dois distritos onde a Rede A poderia inaugurar um ponto de venda.

\section{CONTRIBUIÇÃO TECNOLÓGICO-SOCIAL}

As contribuições das ferramentas de geomarketing e geoinformação são fundamentais para as decisões de varejo. A visualização geoespacial das informações disponibilizadas em tabelas e gráficos permite maior compreensão por parte de gestores, consultores, funcionários e clientes. A técnica de geoprocessamento permite analisar fenômenos e distribuições 
aplicadas ao marketing na medida em que auxiliam a identificar padrões, além de utilizar, simultaneamente, inúmeros bancos de dados.

Este trabalho contribui para ampliar a discussão sobre as ferramentas SIG na área de marketing e para mostrar sua aplicação em um caso real. Além disso, este relato mostra como ferramentas e dados gratuitos podem ser fortes aliados ao negócio.

\section{REFERÊNCIAS}

Aranha, F. (1996). Sistema de Informação Geográfica: uma arma estratégica para o Database Marketing. Revista de Administração de Empresas, 36(2), 12-16.

Baviera-Puig, A., Roig-Tierno, N., Buitrago-Vera, J., \& Mas-Verdu, F. (2013). Comparing trade areas of technology centres using 'Geographical Information Systems'. The Service Industries Journal, 33(7-8), 789-801.

Blackwell, R. D., Miniard, P. W., \& Engel, J. F. (2011). Comportamento do consumidor. São Paulo: Cengage Learning.

Corujo, M. S. (2014). A contribuição do geomarketing para o processo decisório de localização de empresas de varejo: um estudo de caso em uma empresa de vestuário feminino. Dissertação de Mestrado, Pontifícia Universidade Católica, PUC, Rio de Janeiro, Brasil.

Fagundes, A. F. A., Moriguchi, S. N., Vargas, R. T, Santana, E. E. P., \& Sousa, C. V. (2009). A contribuição do geomarketing para a administração de vendas: um estudo de caso em uma empresa de telecomunicações. Anais do Encontro Nacional de Engenharia de Produção, 29, Salvador, BA, Brasil.

Fundação Seade. (s/d). Informações dos municípios paulistas. Recuperado em 31 de outubro, 2016, de http://www.imp.seade.gov.br/frontend/\#/perfil.

Gijsbrechts, E., Campo, K., \& Goossens, T. (2003). The impact of store flyers on store traffic and store sales: a geo-marketing approach. Journal of Retailing, 79(1), 1-16.

Instituto Brasileiro de Geografia e Estatística - IBGE. (2010). Censo demográfico 2010. Recuperado em 28 de junho, 2016, de http://www.censo2010.ibge.gov.br/

Kamakura, W. A., \& Mazzon, J. A. (2013a). Socioeconomic status and consumption in an emerging economy. International Journal of Research in Marketing, 30, 4-18. 
Kamakura, W. A., \& Mazzon, J. A. (2013b). Estratificação socioeconômica e consumo no Brasil. São Paulo: Blucher.

Kotler, P., \& Keller, K. L. (2006). Administração de Marketing (12a ed.). São Paulo: Pearson Prentice Hall.

Machado, C. C., Francisco, E. R., \& Ribeiro, J. G. P. (2006). A geodemografia e os estudos de mercado: identificação de mercados potenciais. In J. R. S. Guimarães (Org.), Demografia dos negócios: campo de estudo, perspectivas e aplicações (vol. 3, pp. 77-95). Campinas: ABEP.

Miller, F. L., Holmes, T. L., \& Mangold, W. G. (2007). Integrating geographic information systems (GIS) into the marketing curriculum. Marketing Education Review, 17 (3), 49-63.

Mittal, V., Kamakura, W. A., \& Govind, R. (2004). Geographic patterns in customer service and satisfaction: an empirical investigation. Journal of Marketing, 68(3), 48-62.

Nunes, A., Santana, C., Bezerra, F., \& Sobral, N. (2014). Knowledge acquisition based on geomarketing information for decision making: a case study on a food company. International Journal of Innovation, Management and Technology, 5(6), 422-427.

Ozimec, A. M., Natter, M., \& Reutterer, T. (2010). Geographical information systems-based marketing decisions: effects of alternative visualizations on decision quality. Journal of Marketing, 74(6), 94-110.

Pozzebon, M., \& Delgado, N. (2012). Participatory Geographic Information Systems in Sierra Nevada, Mexico. Revue international de cas en gestion, 10(1), 19p.

Prochnow, R. M. (2011). Potencial dos dados do setor censitário brasileiro aplicado ao marketing de um fast food delivery. Revista Geográfica de América Central, 2(47E), 1-22.

Thompson, S. (2010, October). Using GIS for local-market penetration, winning strategies. Franchising World, 23-25.

Wedel, M., \& Kamakura, W. A. (2000). Market segmentation: conceptual and methodological foundations. Boston: Kluwer Academic. 